\section{Exercício físico no ganho de peso e resultados perinatais em gestantes com sobrepeso e obesidade: uma revisão sistemática de ensaios clínicos}

\author{
Physical exercise, weight gain, and perinatal \\ outcomes in overweight and obese pregnant \\ women: a systematic review of clinical trials
}

Simony Lira do Nascimento 1

Fernanda Garanhani de Castro Surita 1

Mary Ângela Parpinelli 1

José Guilherme Cecatti 1
${ }_{1}^{1}$ Faculdade de Ciências Médicas, Universidade Estadual de Campinas, Campinas, Brasil.

Correspondência S. L. Nascimento

Departamento de

Tocoginecologia, Faculdade de Ciências Médicas,

Universidade Estadual de Campinas.

Rua Alexander Fleming 101, Campinas, SP 13083-881, Brasil.

simonylira@yahoo.com.br

\section{Abstract}

This study aimed to evaluate the effects of exercise on weight gain and perinatal outcomes among overweight and obese pregnant women, through a systematic review in MEDLINE/ PubMed, EMBASE, LILACS, and SciELO. We included ten clinical trials that evaluated the effectiveness of exercise with or without combined diet in the control of gestational weight gain. Three studies were randomized, and methodological quality was assessed using the CONSORT 2010 Checklist, but none met all the criteria. Four studies showed weight gain differences between groups. The majority (60\%) of studies showed no differences in perinatal outcomes (mode of delivery, gestational age at birth, birth weight). In conclusion, few studies confirmed the positive effect of exercise in controlling weight gain during pregnancy, thus requiring more research in this direction. Regarding perinatal outcomes, mild to moderate exercise does not appear to be decisive for perinatal outcomes, and is safe for pregnant women with overweight and obesity.

Exercise; Pregnant Women; Weight Gain; Obesity

\section{Introdução}

O contínuo aumento da prevalência de obesidade em todo o mundo tem sido considerado uma epidemia global e uma das mais relevantes ameaças à saúde, inclusive em mulheres em idade reprodutiva ${ }^{1}$. A origem deste problema está em hábitos de vida não saudáveis, como dietas hipercalóricas, sedentarismo e o fumo ${ }^{2}$.

Segundo critério da Organização Mundial da Saúde (OMS), a prevalência de obesidade na gestação varia de $1,8 \%$ a 25,3\% 1,2. Em seis capitais brasileiras a obesidade foi encontrada em 5,5\% das gestantes e, quando somadas àquelas com sobrepeso, representaram $25 \%$ de uma coorte de 5.564 mulheres, sendo mais frequente em mulheres mais velhas, negras, com menor grau de escolaridade e multíparas 3 .

Para a definição de obesidade na gestação, o Institute of Medicine dos Estados Unidos (IOM) considera o índice de massa corporal (IMC) prégestacional maior que $30 \mathrm{~kg} / \mathrm{m}^{2}$, considerando uma faixa de ganho de peso adequado durante a gestação para cada categoria de IMC 4 . Outras definições para obesidade durante a gestação são: peso maior que $110 \%$ a $120 \%$ do peso ideal na primeira consulta de pré-natal e peso absoluto da gestante maior que $90,7 \mathrm{~kg} 5$.

A obesidade na gravidez aumenta a morbidade e mortalidade tanto para mãe quanto para o feto no decorrer da gestação e seu desfecho 6 . Durante a gestação e parto, entre as complica- 
ções maternas associadas à obesidade estão: diabetes gestacional, hipertensão gestacional e pré-eclâmpsia, tromboembolismo venoso, necessidade de indução do trabalho de parto e altos índices de cesariana. Também são observadas complicações clínico-cirúrgicas como infecção da ferida cirúrgica, hemorragia, anemia, infecção urinária e endometrite, além de incontinência urinária de esforço, depressão e dificuldades na amamentação 2,6,7.

Também é descrita associação entre obesidade na gestação e resultados neonatais adversos, a exemplo da macrossomia, síndrome metabólica e predisposição à obesidade nas crianças que aparecem secundariamente ao diabetes gestacional, além de defeitos no tubo neural e anomalias congênitas 8,9,10. Concomitantemente, o diagnóstico ultrassonográfico pré-natal é limitado pela interposição do tecido adiposo, que dificulta a visualização do feto por meio de ultrassonografia 11.

Enquanto já estão bem definidos na literatura os impactos negativos da obesidade nos resultados obstétricos e perinatais, ainda são limitadas as informações que esclarecem como tais adversidades poderiam ser minimizadas por intervenções específicas. Dentre as diversas abordagens no manejo da gestante obesa, o exercício físico vem sendo apontado como uma alternativa, porém ainda há controvérsias acerca de sua segurança e seus efeitos durante a gestação ${ }^{2}$.

A atividade física é definida como qualquer movimento corporal voluntário que resulta em gasto energético maior que o gasto em repouso 12. Já o exercício físico é caracterizado como toda atividade física estruturada, planejada e repetitiva que tem por objetivo a melhoria da saúde e a manutenção de um ou mais componentes da aptidão física. Esses termos se confundem e muitas vezes são utilizados como sinônimos 13 .

Ensaios clínicos sugerem mudanças no estilo de vida, adequação da dieta e programas de exercício físico na tentativa de prevenir o excesso de ganho de peso gestacional (GPG), a retenção de peso pós-gestacional e mesmo resultados adversos maternos e perinatais associados à obesidade e ganho de peso excessivo 14,15,16.

Portanto, propõe-se avaliar por meio de uma revisão sistemática de que forma uma intervenção como a prática de exercício físico na gestação interfere no ganho de peso e nos resultados e perinatais (via de parto, idade gestacional ao nascimento, peso do recém-nascido) de gestantes com sobrepeso e obesidade.

\section{Métodos}

Realizou-se uma revisão sistemática com pesquisa bibliográfica da literatura específica utilizando os seguintes termos: (exercise/exercício OR physical activity/atividade física) AND (pregnancy/gravidez OR gestation/gestação) AND (obesity/obesidade OR overweight/sobrepeso), através de pesquisa via Descritores em Ciência da Saúde (DeCS) / Medical Subject Headings (MeSH) nas bases de dados da MEDLINE/PubMed, EMBASE, SciELO e LILACS. Em seguida os principais resultados procurados foram combinados à pesquisa: GPG, hipertensão arterial gestacional, pré-eclâmpsia, diabetes gestacional, via de parto, peso ao nascer, prematuridade, capacidade cardiorrespiratória e qualidade de vida.

Foram considerados critérios de inclusão: artigos realizados com humanos; do tipo ensaio clínico; intervenção envolvendo prática supervisionada e/ou orientações sobre exercício físico; e que contivessem em sua amostra gestantes com sobrepeso e/ ou obesidade; publicados entre 1980 e 2010. Após a busca, todos os artigos identificados foram triados através da leitura dos respectivos títulos e resumos. Os artigos não originais, de revisão, bem como aqueles que não relatavam resultados originais de intervenções (exercício físico) em gestantes obesas ou com sobrepeso, foram excluídos. Os artigos remanescentes que potencialmente tratavam do assunto foram acessados na íntegra para avaliação, de forma independente por dois pesquisadores, que posteriormente confrontaram seus resultados, resolvendo as discrepâncias por consenso.

Após a leitura completa dos estudos procedeu-se a avaliação da qualidade metodológica dos mesmos. Foram considerados adequados os estudos que apresentassem grupo controle, independente da sua forma de alocação, e que demonstrassem resultados objetivos sobre o ganho de peso materno e resultados perinatais. Os que não cumpriam estes critérios foram também excluídos da análise. As listas de referências dos artigos restantes foram checadas manualmente para identificação de outros eventuais estudos que não tivessem sido identificados anteriormente pelo método empregado.

Para avaliação da qualidade metodológica dos ensaios clínicos randomizados incluídos nesta revisão foi utilizado o Consolidated Standards of Reporting Trials (CONSORT Statement) através do CONSORT 2010 Checklist 17, uma lista com 25 itens que permite aos leitores entender os métodos, a análise e a validade dos resultados apresentados pelos ensaios clínicos randomizados. Assim como para o direcionamento desta revisão sistemática foram seguidos os critérios 
do Preferred Reporting Items for Systematic Reviews and Meta-Analyses (PRISMA Statement) 18, uma ferramenta criada para ajudar os autores de revisões sistemáticas ou meta-análises a avaliar os resultados de uma intervenção em saúde, a fim de assegurar uma informação transparente e completa sobre seus resultados.

O estudo seguiu todos os princípios éticos e de confidencialidade de informações que se recomendam, por se tratar de análise de resultados já publicados em outros artigos, não foi necessária a aprovação formal de um comitê de ética em pesquisa.

\section{Resultados}

O processo de pesquisa identificou inicialmente 993 artigos através da busca pelas palavraschave, em seguida estes foram triados utilizando alguns dos critérios de inclusão como filtros de pesquisa: artigos com seres humanos ( $\mathrm{n}=888$ ), estudos publicados entre 1980 e 2010 ( $\mathrm{n}=877$ ), ensaios clínicos $(n=126)$ e depois de lidos os títulos e resumos foram selecionados dez artigos baseados nos critérios de inclusão (Figura 1).

Como descrito na Tabela 1, entre os dez estudos que avaliaram a efetividade do exercício combinado ou não com dieta no controle do GPG, em quatro houve diferença significativa quanto ao melhor controle do GPG no grupo de intervenção 19,20,21,22. Nem todos os estudos avaliaram exclusivamente gestantes com sobrepeso e obesidade, porém esses grupos estavam presentes em todas as amostras.

Os estudos têm métodos variados, com diferentes formas de abordagem. Aqueles em que a intervenção se baseou apenas em educação quanto à dieta, exercício e ganho de peso não obtiveram sucesso 16,23,24,25. Por outro lado, aqueles em que o exercício foi supervisionado apresentaram resultados positivos 19,20,21. Seis estudos não demonstraram diferença significativa entre as gestantes que praticaram exercício físico e as do grupo controle quanto aos resultados perinatais (via de parto, idade gestacional ao nascer, peso do recém-nascido) 19,20,21,22,24,25. Entre os estudos avaliados, a caminhada foi o exercício mais freqüente 16,20,21,26,27 e o exercício aquático foi utilizado em apenas um estudo 19.

Dos dez ensaios clínicos selecionados, três são randomizados e passaram pela avaliação através do CONSORT 2010 Checklist 17 . Sendo que nenhum deles cumpriu todos os critérios (Tabela 2). Os outros sete estudos adotaram metodologias diversas para descrição da alocação no grupo controle 19,20,23,27, ou com controles históricos $21,24,25$. Estes fatores associados à inviabilidade de cegamento para intervenções com exercício físico prejudicam a qualidade metodológica enfraquecendo suas conclusões.

Devido à grande variedade de intervenções (únicas ou combinadas) através de diferentes métodos, incluindo gestantes de baixo risco ou com co-morbidades e idade gestacional diversas, não consideramos apropriada a combinação de resultados para realizar uma meta-análise.

\section{Discussão}

Os resultados obtidos através desta revisão mostraram que existe uma grande limitação em encontrar estudos originais com qualidade metodológica adequada sobre o tema em questão, e principalmente para a população de gestantes obesas. Além disso, o tipo de abordagem desejada, ou seja, realização de exercício físico supervisionado ou não, esbarra na dificuldade do controle deste tipo de intervenção. Assim, são poucos os estudos randomizados, sendo necessário incluir estudos com metodologias diversas para aumentar o conhecimento sobre os resultados.

\section{Indicações do exercício físico em gestantes com sobrepeso e obesidade}

O exercício durante a gestação já faz parte das recomendações do American College of Obstetricians and Gynecologists (ACOG) desde meados da década 90. É reconhecido como uma prática segura, indicado para gestantes saudáveis, desde que respeitando uma intensidade, duração, freqüência e particularidades de cada mulher 28. Em relação à obesidade na gestação a ACOG não se posiciona quanto à segurança do exercício, apenas a obesidade mórbida aparece como uma das contraindicações relativas para tal prática ${ }^{28}$. A execução de um programa de exercícios físicos regulares e um estilo de vida saudável proporciona benefícios durante o período gestacional, reduzindo a incidência de efeitos indesejáveis durante a gravidez como cãibras, edema e fadiga 29,30, além de melhorar a função cardiovascular ${ }^{31}$.

Outros estudos devem ser citados no intuito de certificar a segurança do exercício entre as gestantes com sobrepeso e obesidade.

Santos et al. 26 avaliaram o efeito do treino aeróbico em esteira ou bicicleta $3 \mathrm{x} /$ semana comparado ao relaxamento $1 \mathrm{x} /$ semana em gestantes com sobrepeso e observaram aumento de ventilação e do consumo de oxigênio no grupo que fez exercício, demonstrando melhora da capacidade cardiorrespiratória. 


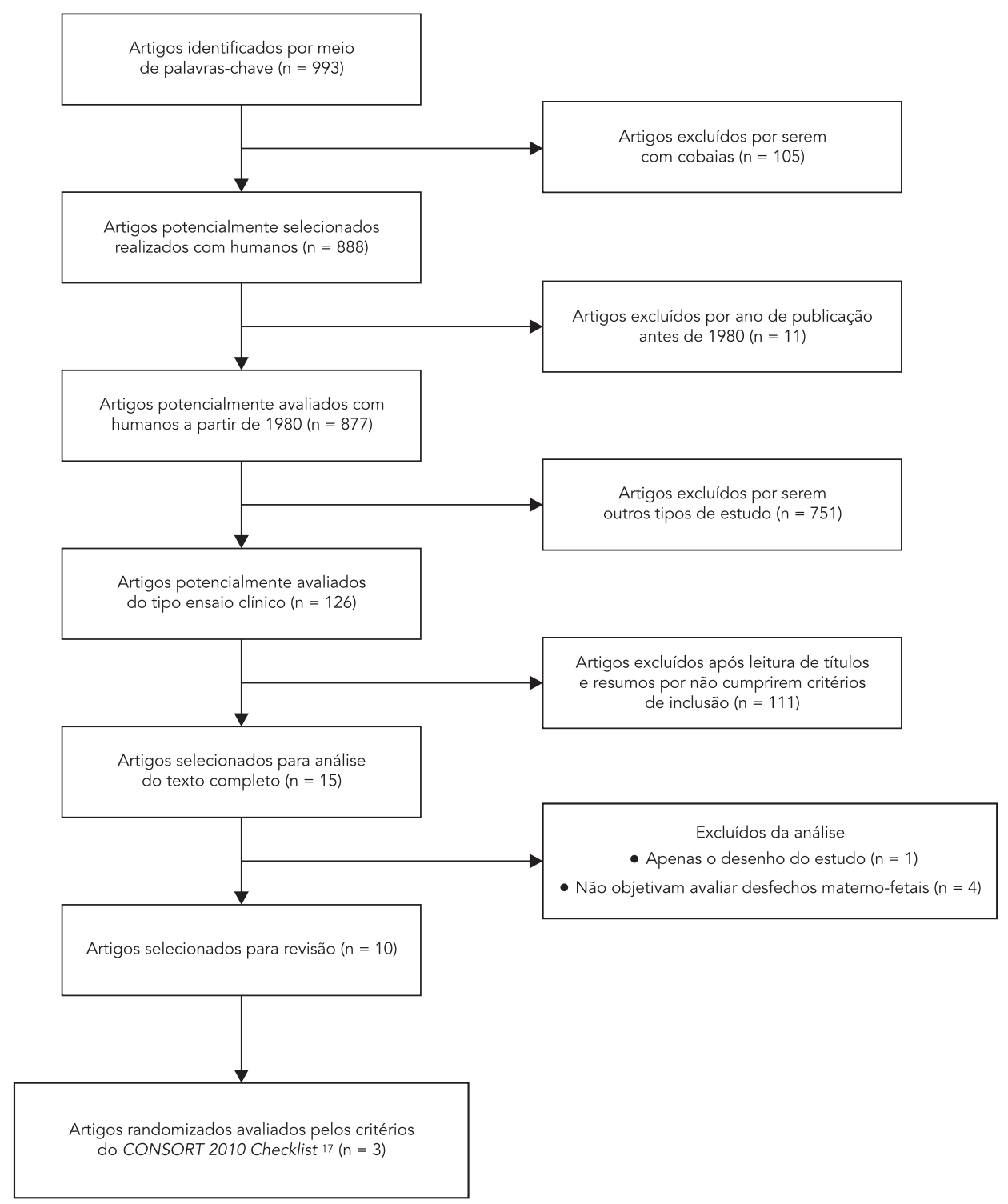

O exercício de intensidade leve a moderada é recomendado a qualquer tipo de gestante, mesmo as sedentárias que desejem iniciar alguma atividade durante a gestação 32 . Neste caso é aconselhado o aumento gradual do tempo e intensidade do exercício 28.

Dentre as atividades aeróbicas praticadas por gestantes, a caminhada mostrou-se a mais popular 33. Quando controlada, a caminhada foi efetiva na prevenção do excesso de ganho de peso na gestação em mulheres com sobrepeso e obesidade, combinada ao controle nutricional 20,21,34.

Um estudo recente comparou gestantes obesas e não obesas através de teste em esteira ergométrica até a fadiga voluntária e concluiu que a resposta ventilatória não foi afetada pela obesidade. Sustentando, assim, a viabilidade do exercício submáximo, como a caminhada, para este grupo 34 . 
Resumo dos estudos envolvendo exercício como intervenção no controle do ganho de peso gestacional em gestantes com sobrepeso e obesidade.

\begin{tabular}{|c|c|c|c|c|}
\hline Autores & Ano & $\begin{array}{l}\text { Características da } \\
\text { população estudada }\end{array}$ & Intervenção & Resultados \\
\hline Gray-Donald et al. 25 & 2000 & $\begin{array}{c}112 \text { no grupo intervenção vs. } 107 \\
\text { controles; mulheres de aldeias Cree } \\
\text { (Quebec, Canadá); todas as faixas } \\
\text { de IMC }\end{array}$ & $\begin{array}{l}\text { Dieta individual, grupos de exercício } \\
\text { e orientações nutricionais através de } \\
\text { meios de comunicação }\end{array}$ & $\begin{array}{c}\text { Sem diferença entre os grupos } \\
\text { quanto a: ganho de peso, taxa de } \\
\text { ganho de peso semanal, glicemia e } \\
\text { peso do recém-nascido }\end{array}$ \\
\hline Polley et al. 16 & 2002 & $\begin{array}{l}57 \text { no grupo intervenção vs. } 53 \\
\text { controles; gestantes de baixa renda; } \\
\qquad I M C>19,8 \mathrm{~kg} / \mathrm{m}^{2}\end{array}$ & $\begin{array}{l}\text { Mudanças de hábito com } \\
\text { informação oral e escrita sobre } \\
\text { ganho de peso adequado, exercício } \\
\text { (estímulo a caminhada, estilo de vida } \\
\text { ativo e alimentação saudável) }\end{array}$ & $\begin{array}{l}\text { Efeito inverso entre as gestantes } \\
\text { com sobrepeso/obesidade: } 59 \% \\
\text { excederam o ganho de peso } \\
\text { adequado no grupo intervenção vs. } \\
32 \% \text { nos controles }\end{array}$ \\
\hline Olson et al. 24 & 2004 & $\begin{array}{l}179 \text { no grupo intervenção vs. } 381 \\
\text { controles; gestantes saudáveis; } \\
\text { IMC normal e sobrepeso }\end{array}$ & $\begin{array}{l}\text { Informações durante o pré-natal } \\
\text { e via e-mail sobre aspectos } \\
\text { nutricionais, exercício e adequação } \\
\text { do ganho de peso }\end{array}$ & $\begin{array}{l}\text { Sem diferença, ganho de peso } \\
\text { adequado: } 41 \% \text { vs. } 45 \% \text { nos } \\
\text { controles. Gestantes de baixa renda } \\
\text { com sobrepeso foram beneficiadas } \\
\text { (72\% vs. } 44 \% \text { nos controles). Sem } \\
\text { diferença nos resultados neonatais }\end{array}$ \\
\hline Santos et al. 26 & 2005 & $\begin{array}{c}37 \text { no grupo intervenção vs. } 35 \\
\text { controles; gestantes de baixo risco; } \\
\text { gestantes com sobrepeso }\end{array}$ & $\begin{array}{l}\text { Avaliar o efeito do exercício na } \\
\text { capacidade cardiorrespiratória; } \\
\text { treino aeróbico (caminhada ou } \\
\text { bicicleta) + exercício de resistência; } \\
\text { controle = relaxamento }\end{array}$ & $\begin{array}{l}\text { Sem diferença quanto ao ganho } \\
\text { de peso, resultados neonatais } \\
\text { e complicações. Aumento da } \\
\text { capacidade cardiorrespiratória no } \\
\text { grupo intervenção }\end{array}$ \\
\hline Claesson et al. 19 & 2007 & $\begin{array}{c}155 \text { no grupo intervenção vs. } 193 \\
\text { controles; gestantes obesas }\end{array}$ & $\begin{array}{l}\text { Restringir o excesso de ganho de } \\
\text { peso }(<7 \mathrm{~kg}) \text {; encontros semanais } \\
\text { com incentivo à mudança de hábitos } \\
\text { e exercícios na água }\end{array}$ & $\begin{array}{c}\text { Gestantes do grupo intervenção } \\
\text { tiveram menor ganho de peso }(8,7 \mathrm{~kg} \\
\text { vs. } 11,3 \mathrm{~kg}) \text { e ganho de peso }<7 \mathrm{~kg} \\
\text { foi maior na intervenção }(35,7 \% \text { vs. } \\
20,5 \%) \text {. Sem diferença nos resultados } \\
\text { neonatais }\end{array}$ \\
\hline Kinnunen et al. 23 & 2007 & $\begin{array}{c}49 \text { no grupo intervenção vs. } 56 \\
\text { controles; primíparas em todas as } \\
\text { faixas de IMC }\end{array}$ & $\begin{array}{c}\text { Aconselhamento sobre dieta e } \\
\text { atividade física (oferecido treino } \\
\text { aeróbico e muscular) }\end{array}$ & $\begin{array}{l}\text { Sem diferença no ganho de peso: } \\
46 \% \text { vs. } 30 \% .15 \% \text { de recém-nascidos } \\
\text { GIG no grupo controle }(p=0,006)\end{array}$ \\
\hline Artral et al. 20 & 2007 & $\begin{array}{c}39 \text { no grupo intervenção vs. } 57 \\
\text { controles; gestantes obesas com } \\
\text { diabetes gestacional }\end{array}$ & $\begin{array}{c}\text { Dieta + exercício (supervisionado } \\
\text { em esteira ou bicicleta) vs. dieta } \\
\text { apenas }\end{array}$ & $\begin{array}{c}\text { Ganho de peso semanal foi } \\
\text { menor no grupo que fez exercício. } \\
50 \% \text { grupo exercitou-se mais de } \\
\text { 150minutos/semana. Sem diferença } \\
\text { nos resultados neonatais }\end{array}$ \\
\hline Davenport et al. 27 & 2008 & $\begin{array}{c}10 \text { no grupo intervenção vs. } 20 \\
\text { controles; gestantes com diabetes } \\
\text { gestacional; IMC > 25kg/m² }\end{array}$ & $\begin{array}{c}\text { Controle da glicemia com } \\
\text { caminhada de baixa intensidade (25 } \\
\text { a } 40 \text { minutos) }\end{array}$ & $\begin{array}{l}\text { Sucesso no controle glicêmico. } \\
50 \% \text { das gestantes nos dois grupos } \\
\text { excederam o ganho de peso } \\
\text { recomendado. Sem diferenças nos } \\
\text { resultados neonatais }\end{array}$ \\
\hline Asbee et al. 22 & 2009 & $\begin{array}{l}57 \text { no grupo intervenção vs. } 43 \\
\text { controles; gestantes de baixo risco; } \\
\text { todas as faixas de IMC (exceto } \\
>40,5 \mathrm{~kg} / \mathrm{m}^{2} \text { ) }\end{array}$ & $\begin{array}{l}\text { Dieta, incentivo exercício } \\
\text { de moderada intensidade e } \\
\text { monitoramento do ganho de peso }\end{array}$ & $\begin{array}{c}\text { Menor ganho de peso na } \\
\text { intervenção }(p=0,01) \text {. Sem } \\
\text { diferença quanto ganho de peso } \\
\text { adequado. Maior taxa de cesariana } \\
\text { por falha de indução nos controles } \\
(58,3 \% \text { vs. } 25 \%)\end{array}$ \\
\hline Mottola et al. 21 & 2010 & $\begin{array}{c}65 \text { no grupo intervenção vs. } 260 \\
\text { controles; gestantes com sobrepeso } \\
\text { e obesidade }\end{array}$ & $\begin{array}{c}\text { Mudança de hábitos nutricionais } \\
\text { e exercício (caminhada } 25 \text { a } 40 \\
\text { minutos) }\end{array}$ & $\begin{array}{l}\text { O ganho de peso foi de } 6,8 \mathrm{~kg} \\
\text { e o ganho de peso semanal foi } \\
0,38 \mathrm{~kg} .80 \% \text { não ganharam peso } \\
\text { excessivamente }\end{array}$ \\
\hline
\end{tabular}

IMC: índice de massa corporal; GIG: grandes para idade gestacional. 
Avaliação da qualidade metodológica dos ensaios clínicos randomizados através do CONSORT 2010 Checklist 17.

\begin{tabular}{|c|c|c|}
\hline Autor & Ano & Itens não cumpridos no CONSORT 2010 Checklist \\
\hline \multirow[t]{4}{*}{ Poley et al. 16} & 2002 & Item 7: não demonstra como foi calculado tamanho amostral \\
\hline & & Itens 8, 9 e 10: não especificam processos da randomização \\
\hline & & Item 14: não define o período da coleta de dados \\
\hline & & Item 19: não registra a ocorrência ou não de efeitos adversos da intervenção \\
\hline Santos et al. 26 & 2005 & Item 7: não demonstra como foi calculado tamanho amostral \\
\hline Asbee et al. 22 & 2009 & Item 19: não registra a ocorrência ou não de efeitos adversos da intervenção \\
\hline
\end{tabular}

No estudo de Hulens et al. 35 verificou-se que mulheres obesas em geral têm estilo de vida sedentário e, quando comparadas as obesas fisicamente ativas, as sedentárias têm pior qualidade de vida relacionada à saúde. Dados sobre atividade física e qualidade de vida em gestantes obesas são raros 2,35.

Conforme citado anteriormente, apesar do alto percentual de gestantes com sobrepeso e obesidade no Brasil 4, a prevalência de atividade física de lazer entre as grávidas brasileiras é baixa. Domingues \& Barros 33 verificaram que apenas 4,7 \% são ativas durante toda a gestação e concluíram que embora a atividade física não seja percebida como prejudicial à gravidez, e as diretrizes nacionais atuais a recomendem, a população parece não mudar de comportamento, por isso deve-se encorajar que mulheres grávidas e as que pretendem engravidar tenham estilo de vida ativo.

Outro fator relacionado com o sobrepeso e o ganho de peso durante a gestação são as queixas de lombalgia, dor pélvica posterior, sensação de peso e formigamento nos membros gerados pelas alterações no sistema músculo-esquelético. Estas alterações podem resultar em limitações das atividades diárias. A prevalência de queixas álgicas nas regiões lombar e pélvica durante a gestação relatada em diferentes estudos varia de $24 \%$ a $90 \% 36$. Essas alterações têm maiores repercussões nas gestantes obesas pela maior sobrecarga no seu sistema músculo-esquelético, dados confirmados em estudo que demonstrou que maior peso e maior IMC pré-gestacional e/ ou gestacional estão relacionados com alta prevalência de lombalgia durante e até seis meses após a gestação caracterizando mais uma indicação para prática de exercício nesta população 37 .

\section{O exercício no controle do ganho de peso gestacional}

A limitação do ganho de peso de gestantes obesas de acordo com as recomendações do IOM (Tabela 3) tem sido apontada como benéfica em diminuir o risco de resultados materno-fetais adversos. Gestantes com sobrepeso e obesidade que ganharam menos de $8 \mathrm{~kg}$ durante a gestação tiveram menores taxas de recém-nascidos grandes para idade gestacional (GIG), pré-eclâmpsia, cesárea e parto vaginal operatório quando comparadas às gestantes que tiveram alto ganho de peso gestacional 38 .

Recomenda-se evitar a perda de peso ou ganho menor do que $5 \mathrm{~kg}$ durante a gestação tendo em vista a associação com trabalho de parto prematuro e recém-nascidos pequenos para idade gestacional (PIG), porém esta associação não foi demonstrada em mulheres com IMC > $26 \mathrm{~kg} / \mathrm{m}^{2} 39$. Nas gestantes diabéticas tratadas com dieta e exercício houve redução significativa na incidência de macrossomia e recém-nascidos PIG 40.

O ganho ponderal fisiológico em uma gestação normal corresponde ao crescimento fetal, líquido amniótico, placenta, útero, tecido mamário e volume sanguíneo aumentados, além do acúmulo variável do líquido tecidual e tecido adiposo. Este último ocorre em função de fatores pré-gestacionais ou demográficos, condições de saúde e fatores comportamentais, como ingestão calórica, exercício físico, tabagismo e orientações quanto a GPG segundo o IOM. Os fatores comportamentais correspondem apenas a $11 \%$ para o risco de ganho de peso excessivo, porém merecem destaque por serem potencialmente modificáveis 41 .

Na última década diversos estudos avaliaram a combinação de intervenções envolvendo atividade física com objetivo de controlar o GPG (Tabela 1). Dentre os estudos que avaliaram a 
Ganho ponderal recomendado para gestantes de acordo com o índice de massa corporal (IMC) pré-gestacional segundo o Institute of Medicine dos Estados Unidos 42.

\begin{tabular}{lcc}
\hline IMC pré-gestacional $\left(\mathbf{k g} / \mathrm{m}^{2}\right)$ & $\begin{array}{c}\text { Ganho de peso }(\mathrm{kg}) \text { semanal médio } \\
\text { no 2o e 3o trimestres de gestação }\end{array}$ & $\begin{array}{c}\text { Ganho de peso }(\mathrm{kg}) \text { total na gestação } \\
\text { Baixo peso }(<18,5)\end{array}$ \\
Adequado $(18,5-24,9)$ & $0,45(0,45-0,59)$ & $12,5-18,0$ \\
Sobrepeso $(25,0-29,9)$ & $0,28(0,23-0,31)$ & $11,5-16,0$ \\
Obesidade $(\leq 30,0)$ & $0,23(0,18-0,28)$ & $7,0-11,5$ \\
\hline
\end{tabular}

combinação de fatores como dieta e exercício, quatro encontram diferença significativa quanto ao ganho ponderal 19,20,21,22.

Programas educativos de estímulo a atividade física não foram efetivos em prevenir o excesso de GPG 16,23,24,25. Entretanto, Asbee et al. 22, com um programa de monitoramento do ganho de peso, dieta e incentivo a um estilo de vida ativo, obtiveram sucesso em controlar o ganho de peso no grupo que recebeu a intervenção, porém quanto ao ganho de peso excessivo a diferença não foi significativa. Esses resultados nos fazem considerar que apenas a orientação às gestantes não é suficiente para a obtenção de controle ponderal com resultados significativos.

Os estudos em que o exercício foi supervisionado e controlado relataram sucesso com a intervenção. Artral et al. 20 introduziram sessões de caminhada ou bicicleta estacionária em intensidade moderada ( $60 \%$ do VO2 máximo), podendo ser uma vez na semana no laboratório e mais seis dias na semana sem supervisão combinados com dieta específica para gestantes obesas diabéticas. O grupo que recebeu essa intervenção teve maior taxa de gestantes que mantiveram ou perderam peso após o início do exercício $446,2 \%$ vs. $21,1 \%)$ e menor média de ganho de peso semanal $(0,1 \pm 0,4 \mathrm{~kg} / \mathrm{semanas} v s .0,3 \pm 0,4 \mathrm{~kg} / \mathrm{se}-$ mana) em relação ao grupo submetido à dieta apenas 20 .

Da mesma forma, por meio de exercício supervisionado, Claesson et al. 19 também obtiveram resultados positivos estudando gestantes obesas. O grupo que recebeu a intervenção, baseada em encontros semanais de incentivo a mudança de hábitos e classes de exercícios aquáticos uma a duas vezes na semana, ganhou menos peso $(8,7 \mathrm{~kg} v s .11,3 \mathrm{~kg})$ e teve maior taxa de gestantes que ganharam menos que $7 \mathrm{~kg}(35,7 \%$ vs. 20,5\%) 19 .

Dois estudos utilizaram protocolos similares introduzindo a caminhada de baixa intensida- de controlada através de contadores de passos, inicialmente com 20 minutos aumentando 2 minutos a cada semana até 40 minutos. Devenport et al. 27 fizeram um estudo piloto com gestantes obesas diabéticas, e apesar do sucesso no controle glicêmico verificaram $50 \%$ de sucesso em prevenir o excesso de ganho de peso gestacional em ambos os grupos. Mottola et al. 21 consideraram sucesso na intervenção com uma taxa de $80 \%$ das gestantes que não ultrapassaram o ganho de peso recomendado a partir do início do programa. Estes estudos demonstram a necessidade de instrumentos simples, de fácil aplicabilidade e entendimento pelas gestantes, aumentando a adesão ao exercício e levando a resultados consistentes.

Para esta população, estes resultados demonstram que intervenções específicas precisam ser baseadas em mudança de comportamento, pois apenas aumentar o conhecimento através de programas de educação parece não ser suficiente, sendo necessária uma intervenção individualizada de nutrição e exercício.

Entretanto, a comparação entre os estudos se torna difícil pelos diferentes parâmetros para avaliar o excesso de GPG. Até a última revisão do IOM em 2009, não era estabelecido um limite superior para o ganho de peso em gestantes obesas, o estudo de Mottola et al. ${ }^{21}$ então, considerou como excesso, ganho de peso $>11,5 \mathrm{~kg}$, o limite superior da faixa de sobrepeso e observou uma taxa de $80 \%$ de gestantes que não excederam este limite. Já outros estudos que não observaram diferença significativa consideraram o limite de $6,8 \mathrm{~kg} 16,19,23,42$.

Ademais, dois estudos em que a amostra foi composta por gestantes em todas as faixas de IMC, observaram menor adesão às recomendações do IOM entres as gestantes com sobrepeso e obesidade. No estudo de Polley et al. 16 as gestantes com IMC $>26 \mathrm{~kg} / \mathrm{m}^{2}$ tiveram um resultado inverso, 59\% das gestantes no grupo intervenção 
excederam o ganho de peso adequado contra $32 \%$ no grupo controle. Asbee et al. 22 também observaram baixas taxas de aderência as recomendações do IOM entre as gestante obesas $(33,3 \%)$ e com sobrepeso (30\%).

Estas controvérsias podem ser atribuídas tanto aos métodos utilizados para análise do peso, que não levam em conta a composição corporal materna, como à dificuldade na adesão das gestantes às orientações quanto à realização de exercício e estilo de vida ativo, reforçado ainda pelo antigo apelo popular de que a gravidez é um estado de reclusão e repouso.

Assim, o ganho de peso durante a gestação é um forte determinante da retenção peso pósparto, por isso, a prevenção do ganho de peso excessivo durante a gestação é importante para evitar o desenvolvimento de sobrepeso e obesidade em mulheres em idade fértil.

\section{O exercício e os resultados perinatais}

Os efeitos do exercício têm sido relatados como favoráveis tanto durante o trabalho de parto como no parto. A obesidade na gestação e o ganho de peso materno estão diretamente relacionados com recém-nascidos GIG e macrossômicos. Estudo observou que recém-nascidos de mães obesas apresentam maior percentual de gordura corporal e massa gorda, explicado pelas alterações metabólicas maternas principalmente no início da gravidez afetando o crescimento feto-placentário e o metabolismo no ambiente uterino, sendo a obesidade o maior responsável pelo aumento nas taxas de GIG diante da sua maior prevalência em relação ao diabetes gestacional 8,9.

Os estudos apresentados na Tabela 1 não avaliaram o trabalho de parto, porém quanto ao peso do recém-nascido a maioria não encontrou diferença considerando a realização ou não de exercício na gestação. Apenas Kinunem et al. 23 demonstraram uma diferença significativa, com taxa de $15 \%$ de recém-nascidos GIG no grupo controle. Assim, com relação aos resultados perinatais, apesar da limitação metodológica, a realização de exercício físico em intensidade leve a moderada, aparentemente, não se relaciona com alterações do peso ao nascimento.

Da mesma forma, a prática de exercício físico na gestação não interfere na via de parto. Con- siderando o tipo de parto, o risco de cesariana aumenta cerca de $7 \%$ a cada unidade aumentada no IMC 43 . Em estudo realizado no Brasil, as taxas de cesárea aumentaram linearmente de acordo com o IMC pré-gestacional, ocorrendo em $24,5 \%$ nas mulheres de baixo peso, $35,1 \%$ nas com peso normal, $43,1 \%$ nas pré-obesas e em $53,2 \%$ nas obesas 44. O estudo de Asbee et al. 22 encontrou maior taxa de cesáreas devido a falha na indução (58,3\%) no grupo controle em comparação a $25 \%$ do grupo submetido à intervenção. As taxas de cesariana em gestantes obesas relacionam-se com a presença de sofrimento fetal, desproporção céfalo-pélvica e antecedente de parto por cesariana 45.

Se por um lado esses resultados sugerem que a realização de exercícios é segura no sentido de não induzir a baixo peso ao nascer e prematuridade, como por muito tempo foi questionado, por ou outro, ainda fica a dúvida se essa intervenção é efetiva em diminuir as taxas de recém-nascidos GIG em gestantes de risco como afirmado através de estudos de coorte.

\section{Considerações finais}

Apesar de parecer consenso que o exercício de intensidade leve a moderada consiste em fator de proteção para alguns desfechos na gravidez, os estudos são controversos sobre o efeito do exercício no controle do ganho de peso. Quanto à via de parto e peso do recém-nascido, o exercício em intensidade leve a moderada perece não ser determinante.

A indicação do exercício físico na gravidez, inclusive para as gestantes obesas, já é considerada uma prática segura, devendo ser conhecida e orientada por toda equipe de profissionais da saúde. Porém, novos estudos devem ser desenvolvidos com objetivo de preencher as lacunas sobre recomendações de intensidade, frequência e duração do exercício, a partir de métodos diretos de avaliação do bem-estar fetal.

Vale ressaltar que a gravidez é um período ideal para a modificação de comportamentos, com isso a introdução de um estilo de vida ativo para pessoas previamente sedentárias se torna interessante, inclusive pela manutenção de efeitos em longo prazo. 


\section{Resumo}

Esta revisão objetivou avaliar os efeitos do exercício físico no ganho de peso e nos resultados perinatais de gestantes com sobrepeso e obesidade, através de revisão sistemática da literatura em bases de dados específicas: MEDLINE/PubMed, EMBASE, SciELO e LILACS. Foram incluídos dez ensaios clínicos que avaliaram a efetividade do exercício combinado ou não com dieta no controle do ganho de peso gestacional. Três estudos são randomizados e a qualidade metodológica foi avaliada através do CONSORT 2010 Checklist, porém nenhum deles cumpriu todos os critérios. Quatro estudos obtiveram diferença quanto ao ganho de peso entre os grupos. A maioria dos estudos (60\%) não demonstrou diferença quanto aos resultados perinatais (via de parto, idade gestacional ao nascer, peso do recémnascido). Poucos estudos confirmam o efeito positivo do exercício no controle do ganho de peso gestacional, necessitando mais pesquisas neste sentido. O exercício em intensidade leve a moderada parece não ser determinante nos resultados perinatais, sendo uma prática segura para gestantes com sobrepeso e obesidade.

Exercício; Gestantes; Ganho de Peso; Obesidade

\section{Colaboradores}

S. L. Nascimento realizou a pesquisa nas bases de dados, participou da seleção dos artigos e da produção do texto e aprovou a versão final. F. G. C. Surita colaborou na seleção dos artigos incluídos na revisão, na produção do texto e aprovação da versão final. M. A. Parpinelli contribuiu na produção do texto e a idealização da revisão, bem com aprovou a versão final. J. G. Cecatti participou da avaliação da qualidade metodológica do artigo, colaborou com texto e aprovou a versão final.

\section{Referências}

1. World Health Organization. Diet nutrition and prevention diseases. Report of Joint WHO/FAO Expert Consultation. Geneva: World Health Organization; 2002. (WHO Technical Report Series, 916).

2. Guelinckx I, Devlieger R, Beckers K, Vansant G. Maternal obesity: pregnancy complications, gestational weight gain and nutrition. Obes Rev 2008; 9:140-50.

3. Nucci LB, Schmidt MI, Duncan BB, Fuchs SC, Fleck ET, Britto MM. Nutritional status of pregnant women: prevalence and associated pregnancy outcomes. Rev Saúde Pública 2001; 35:502-7.

4. Institute of Medicine. Nutrition during pregnancy. Washington DC: National Academic Press; 1990.

5. Hall LF, Neubert AG. Obesity and pregnancy. Obstet Gynecol Surv 2005; 60:253-60.

6. Rouse DJ, Nuthalapaty FS. The impact of obesity on fertility and pregnancy. UpToDate 2007. http:// www.uptodate.com (acessado em 20/Mar/2010).

7. Li R, Jewell S, Grummer-Strawn L. Maternal obesity and breast-feeding practices. Am J Clin Nutr 2003; 77:931-6.

8. Catalano PM. Obesity and pregnancy: the propagation a vicious cicle? J Clin Endocrinol Metab 2003; 88:3505-6.
9. Catalano PM, Ehrenberg HM. The short- and long-term implications of maternal obesity on the mother and her offspring. BJOG 2006; 113:1126-33.

10. Stothard KJ, Tennant PW, Bell R, Rankin J. Maternal overweight and obesity and the risk of congenital anomalies: a systematic review and meta-analysis. JAMA 2009; 301:636-50.

11. LaCoursiere DY, Bloebaum L, Duncam JD, Vaner MW. Population-based trends and correlation of maternal overweight and obesity, Utah 1991-2001. Am J Obstet Gynecol 2005; 192:832-9.

12. United States Department of Health and Human Services/Centers for Disease Control and Prevention/National Center for Chronic Disease Prevention and Health Promotion/President's Council on Physical Fitness and Sports. Physical activity and health: a report of the Surgeon General Executive Summary. Atlanta: National Center for Chronic Disease Prevention and Health Promotion; 1996.

13. Caspersen CJ, Powell KE, Christenson GM. Physical active, exercise and physical fitness: definition and distinction for health-related research. Public Health Rep 1985; 100:126-31. 
14. Weissgerber TL, Wolfe LA, Davies GA, Mottola MF. Exercise in the prevention and treatment of maternal-fetal disease: a review of the literature. Appl Physiol Nutr Metab 2007; 31:661-74.

15. O’Toole ML, Sawicki MA, Artal R. Structured diet and physical activity prevent postpartum weight retention. J Women's Health 2003; 12:991-8.

16. Polley BA, Wing RR, Sims CJ. Randomized controlled trial to prevent excessive weight gain in pregnant women. Int J Obesity 2002; 26:1494-502.

17. Schulz KF, Altman DG, Moher D; CONSORT Group. CONSORT 2010 statement: updated guidelines for reporting parallel group randomised trials. Ann Int Med 2010; 152:726-32.

18. Moher D, Liberati A, Tetzlaff J, Altman DG; PRISMA Group. Preferred reporting items for systematic reviews and meta-analyses: the PRISMA statement. BMJ 2009; 339:b2535.

19. Claesson I-M, Sydsjo G, Brynhildsen J, Cedergren $\mathrm{M}$, Jeppsson A, Nistron F, et al. Weight gain restriction for obese pregnant women: a case-control intervention study. BJOG 2008; 115:11-50.

20. Artral R, Catazaro RB, Gavard JA, Mostello DJ, Friganza JC. A lifestyle intervention of weight-gain restriction: diet and exercise in obese women with gestational diabetes mellitus. Appl Physiol Nutr Metab 2007; 32:596-601.

21. Mottola MF, Giroux I, Gratton R, Hammond J, Hanley A, Harris S, et al. Nutrition and exercise prevent excess weight gain in overweight pregnant women. Med Sci Sports Exerc 2010; 42:265-72.

22. Asbee SM, Jenkins TR, Butler JR, White J, Elliot M, Rutledge A. Preventing excessive weight gain during pregnancy through dietary and lifestyle counseling: a randomized controlled trial. Obstet Gynecol 2009; 113(2 Pt 1):305-12.

23. Kinnunen TI, Pasanen M, Fogelholm M, HilakiviClarke L, Weiderpass E, Luoto R. Preventing excessive weight gain during pregnancy: a controlled trial in primary health care. Eur J Clin Nutr 2007; 61:884-91.

24. Olson CM, Strawderman MS, Reed RG. Efficacy of an intervention to prevent excessive gestational weight gain. Am J Obstet Gynecol 2004; 191:530-6.

25. Gray-Donald K, Robinson E, Collier A, David K, Renaud L, Rodrigues S. Intervening to reduce weight gain in pregnancy and gestational diabetes mellitus in Cree communities: an evaluation. CMAJ 2000; 163:1247-51.

26. Santos IA, Stein R, Fuchs SC, Duncan BB, Ribeiro J, Koeff LR, et al. Aerobic exercise and submaximal functional capacity in overweight pregnant women: a randomized trial. Obstet Gynecol 2005; 106:243-9.

27. Davenport MH, Mottola MF, McManus R, Gratton R. A walking intervention improes capillary glucose control in women with gestational diabetes mellitus: a pilot study. Appl Physiol Nutr Metab 2008; 33:511-7.

28. ACOG Committee Obstetric Practice. ACOG Committee opinion. Number 267, January 2002: exercise during pregnancy and the postpartum period. Obstet Gynecol 2002; 99:171-3.

29. Wolfe AL, Davies LAG. Canadian guidelines for exercise in pregnancy. Clin Obstet Gynecol 2003; 46:488-93.
30. Dempsey CJ, Butler LC, Williams AM. No need for a pregnant pause: physical activity may reduce the occurrence of gestational diabetes mellitus and pre-eclampsia. Exerc Sport Sci Rev 2005; 33:141-9.

31. Clapp JF. Exercise and pregnancy: a clinical update. Clin Sports Med 2000; 19:273-86.

32. Arena B, Maffulli N. Exercise in pregnancy: how safe is it? Sports Medicine \& Arthroscopy Review 2002; 10:15-22.

33. Domingues MR, Barros AJD. Leisure-time physical activity during pregnancy in the 2004 Pelotas Birth Cohort Study. Rev Saúde Pública 2007; 41:173-80.

34. Devenport MH, Steinback CD, Mottola MF. Impact of pregnancy and obesity on cardiorespiratory responses during weight-bearing exercise. Respir Physiol Neurobiol 2009; 167:341-7.

35. Hulens M, Vansanst G, Claessens AL, Lysens R, Muls E, Rezewinick R. Health-related quality of life in physically active and sedentary obese women. Am J Hum Biol 2002; 14:777-85.

36. Mogren I, Pohjanen A. Low back pain and pelvic pain during pregnancy: prevalence and risk factors. Spine (Phila Pa 1976) 2005; 30:983-91.

37. Mogren IM. BMI, pain and hyper-mobility are determinants of long-term outcome for women with low back pain and pelvic pain during pregnancy. Eur Spine J 2006; 15:1093-102.

38. Cedergren MI. Effects of gestacional weight gain and body mass index on obstetric outcomes in Sweden. Int J Gynecol Obstet 2006; 93:269-74.

39. Rae A, Bond D, Evans SF, North F, Roberman B, Walters B. A randomized controlled trial of dietary energy restrictionin management of obese women with gestacional diabetes. Aust N Z J Obstet Gynaecol 2000; 40:416-22.

40. Crowther CA, Hiller JE, Moss JR, McPhee AJ, Jeffries WS, Robinson JS, et al. Effect of treatment of gestacional diabets mellitus on pregnancy outcomes. $\mathrm{N}$ Eng J Med 2005; 352:2477-86.

41. Brawarsky P, Stoland NE, Jackson RA, Fuentes-Afflick E, Escobar GJ, Rubashkin N, et al. Pre-pregnancy and pregnancy-related factors and the risk of excessive or inadequate gestational weight gain. Int J Gynaecol Obstet 2005; 91:125-31.

42. Institute of Medicine. Weight gain during pregnancy: reexamining the guidelines. http://www. iom.edu/pregnancyweightgain (acessado em 10/ Mai/2010).

43. Galtier-Dereure F, Boegner C, Bringer J. Obesity and pregnancy: complications and cost. Am J Clin Nutr 2000; 71(5 Suppl):1242S-8S.

44. Seligman LC, Duncan BB, Branchtein L, Gaio DSM, Mengue SS, Schmidt MI. Obesity and gestational weight gain: cesarean delivery and labor complications. Rev Saúde Pública 2006; 40:457-65.

45. Bianco AT, Smilen SW, Davis Y, Lopez S, Lapinski R, Lockwood CJ. Pregnancy outcome and weight gain recommendations for the morbidly obese woman. Obstet Gynecol 1998; 91:97-102.

Recebido em 08/Jun/2010

Versão final reapresentada em 15/Out/2011 Aprovado em 14/Jan/2011 